\title{
Uniform Tail Asymptotics for the Stochastic Present Value of Aggregate Claims in the Renewal Risk Model
}

\author{
Qihe Tang $^{[a]}$, Guojing Wang ${ }^{[b], *}$, and Kam C. Yuen ${ }^{[c]}$ \\ ${ }^{[a]}$ Department of Statistics and Actuarial Science, The University of Iowa \\ 241 Schaeffer Hall, Iowa City, IA 52242, USA \\ E-mail: qtang@stat.uiowa.edu \\ [b] Department of Mathematics, Suzhou University \\ Suzhou 215006, PR China \\ E-mail: wangguojing@hotmail.com \\ [c] Department of Statistics and Actuarial Science, The University of Hong Kong \\ Pokfulam Road, Hong Kong \\ E-mail: kcyuen@hku.hk
}

December 9, 2009

\begin{abstract}
Consider an insurer who is allowed to make risk-free and risky investments. The price process of the investment portfolio is described as a geometric Lévy process. We study the tail probability of the stochastic present value of future aggregate claims. When the claim-size distribution is of Pareto type, we obtain a simple asymptotic formula which holds uniformly for all time horizons. The same asymptotic formula holds for the finite- and infinite-time ruin probabilities. Restricting our attention to the so-called constant investment strategy, we show how the insurer adjusts his investment portfolio to maximize the expected terminal wealth subject to a constraint on the ruin probability.

Keywords: Asymptotics; Constant investment strategy; Lévy process; Portfolio optimization; Regular variation; Ruin probability; Uniformity.

Mathematics Subject Classification: Primary 91B30; Secondary 60G51, 60K05, 91B28
\end{abstract}

\section{Introduction}

Consider the renewal risk model in which successive claims, $X_{1}, X_{2}, \ldots$, form a sequence of independent, identically distributed (i.i.d.), and nonnegative random variables with generic

\footnotetext{
${ }^{*}$ Corresponding author: Guojing Wang.
} 
random variable $X$ and common distribution $F$ on $[0, \infty)$, and their arrival times, $0 \leq \tau_{1} \leq$ $\tau_{2} \leq \cdots$, constitute a renewal counting process

$$
N_{t}=\#\left\{n=1,2, \ldots: \tau_{n} \leq t\right\}, \quad t \geq 0 .
$$

For later use, we write $\tau_{0}=0$ and $\tau_{1}=\tau$. To avoid triviality, throughout the paper, we assume that $\tau$ is a nonnegative random variable non-degenerate at 0 . The amount of aggregate claims up to time $t$ appears to be a compound sum of the form

$$
S_{t}=\sum_{k=1}^{N_{t}} X_{k}, \quad t \geq 0
$$

where the summation over an empty set of indices produces a value of 0 .

Suppose that the insurer is allowed to make risk-free and risky investments. The price process of the investment portfolio is described as a geometric Lévy process $\left\{\mathrm{e}^{R_{t}}, t \geq 0\right\}$; that is to say, $\left\{R_{t}, t \geq 0\right\}$ is a Lévy process which starts with 0 , has independent and stationary increments, and is stochastically continuous. This assumption on price processes is widely used in mathematical finance. We refer the reader to the monograph of Cont and Tankov (2004) and a recent survey paper of Paulsen (2008). See also Paulsen (1993, 2002), Paulsen and Gjessing (1997), Wang and Wu (2001), Kalashnikov and Norberg (2002), Cai (2004), and Yuen et al. (2004, 2006), among others. For general theory of Lévy processes, see Sato (1999), Cont and Tankov (2004), and Applebaum (2004).

As usual, we assume that all sources of randomness, $\left\{X_{1}, X_{2}, \ldots\right\},\left\{N_{t}, t \geq 0\right\}$, and $\left\{R_{t}, t \geq 0\right\}$, are mutually independent. The stochastic present value of future aggregate claims up to time $t$ can be expressed as

$$
D_{t}=\int_{0-}^{t} \mathrm{e}^{-R_{s-}} \mathrm{d} S_{s}=\sum_{k=1}^{\infty} X_{k} \mathrm{e}^{-R_{\tau_{k}}} 1_{\left(\tau_{k} \leq t\right)}, \quad t \geq 0 .
$$

In this paper, we shall focus on the tail probability of $D_{t}$ and aim at a simple asymptotic formula which holds uniformly for all time horizons. We shall also pursue applications of our result to ruin theory.

The rest of the paper consists of three sections. Section 2 presents our first main result after recalling some preliminaries; Section 3 shows applications of this result to the calculation of the finite- and infinite-time ruin probabilities and to a portfolio optimization problem with a constraint on the finite-time ruin probability; and Section 4 proves the results after presenting a series of lemmas.

\section{Preliminaries and Main Result}

Throughout the paper, we assume that the Lévy process $\left\{R_{t}, t \geq 0\right\}$ in (1.2) is right continuous with left limit with Lévy triplet $\left(r, \sigma^{2}, \rho\right)$, where $-\infty<r<\infty, \sigma \geq 0$ are two constants and $\rho$ is a measure on $(-\infty, \infty)$, called Lévy measure, satisfying $\rho(\{0\})=0$ and 
$\int_{-\infty}^{\infty}\left(y^{2} \wedge 1\right) \rho(\mathrm{d} y)<\infty$. Let $\mathrm{E} R_{1}>0$, so that $R_{t}$ drifts to $\infty$ almost surely as $t \rightarrow \infty$. The Laplace exponent for $\left\{R_{t}, t \geq 0\right\}$ is defined as

$$
\phi(z)=\log \mathrm{Ee}^{-z R_{1}}, \quad z \in(-\infty, \infty) .
$$

If $\phi(z)$ is finite, then

$$
\phi(z)=\frac{1}{2} \sigma^{2} z^{2}-r z+\int_{-\infty}^{\infty}\left(\mathrm{e}^{-z y}-1+z y 1_{(-1,1)}(y)\right) \rho(\mathrm{d} y)
$$

and

$$
\mathrm{Ee}^{-z R_{t}}=\mathrm{e}^{t \phi(z)}<\infty, \quad t \geq 0 ;
$$

see, for example, Proposition 3.14 of Cont and Tankov (2004). We refer the reader to Cont and Tankov (2004) and Klüppelberg and Kostadinova (2008) for explicit expressions for the Laplace exponent $\phi(\cdot)$ for some commonly-used Lévy processes.

Recall the renewal function of the renewal counting process $\left\{N_{t}, t \geq 0\right\}$, defined as

$$
\lambda_{t}=\mathrm{E} N_{t}=\sum_{k=1}^{\infty} \operatorname{Pr}\left(\tau_{k} \leq t\right), \quad t \geq 0 .
$$

In particular, if $\left\{N_{t}, t \geq 0\right\}$ is a Poisson process with intensity $\lambda>0$, then $\lambda_{t}=\lambda t$; and if $\tau$ follows a $\Gamma(2, \lambda)$ distribution, then $\lambda_{t}=\lambda t / 2-\left(1-\mathrm{e}^{-2 \lambda t}\right) / 4$. More general examples of the renewal counting process $\left\{N_{t}, t \geq 0\right\}$ allowing explicit forms of the renewal function $\lambda_{t}$ can be found in Asmussen (2003, pages 88 and 148).

Denote by $\Lambda$ the set of all $t$ for which $0<\lambda_{t} \leq \infty$. With $\underline{t}=\inf \{t: \operatorname{Pr}(\tau \leq t)>0\}$, it is clear that

$$
\Lambda= \begin{cases}{[\underline{t}, \infty],} & \text { if } \operatorname{Pr}(\tau=\underline{t})>0 \\ (\underline{t}, \infty], & \text { if } \operatorname{Pr}(\tau=\underline{t})=0\end{cases}
$$

For notational convenience, we write $\Lambda_{T}=[0, T] \cap \Lambda$ for every fixed $T \in \Lambda$.

We shall assume that the claim-size distribution $F$ is regularly-varying tailed, hence heavy tailed; that is, $\bar{F}(x)=1-F(x)>0$ holds for all $x \geq 0$ and there is some constant $\alpha$, $0<\alpha<\infty$, such that the relation

$$
\lim _{x \rightarrow \infty} \frac{\bar{F}(x y)}{\bar{F}(x)}=y^{-\alpha}
$$

holds for all $y>0$. We use $F \in \mathcal{R}_{-\alpha}$ to signify the regularity property in (2.3) and use $\mathcal{R}$ to denote the union of all $\mathcal{R}_{-\alpha}$ over the range $0<\alpha<\infty$. The class $\mathcal{R}$ contains a lot of popular distributions such as Pareto, Burr, Loggamma, and $t$ distributions.

Hereafter, all limit relationships are for $x \rightarrow \infty$ unless stated otherwise. For two positive functions $a(\cdot)$ and $b(\cdot)$, we write $a(x) \lesssim b(x)$ if $\lim \sup a(x) / b(x) \leq 1, a(x) \gtrsim b(x)$ if $\liminf a(x) / b(x) \geq 1$, and $a(x) \sim b(x)$ if both. Furthermore, for two positive bivariate functions $a(\cdot, \cdot)$ and $b(\cdot, \cdot)$, we say that the asymptotic relation $a(x, t) \sim b(x, t)$ holds uniformly for $t$ in a nonempty set $\Delta$ if

$$
\lim _{x \rightarrow \infty} \sup _{t \in \Delta}\left|\frac{a(x, t)}{b(x, t)}-1\right|=0
$$


Clearly, the asymptotic relation $a(x, t) \sim b(x, t)$ holds uniformly for $t \in \Delta$ if and only if

$$
\limsup _{x \rightarrow \infty} \sup _{t \in \Delta} \frac{a(x, t)}{b(x, t)} \leq 1 \quad \text { and } \quad \liminf _{x \rightarrow \infty} \inf _{t \in \Delta} \frac{a(x, t)}{b(x, t)} \geq 1
$$

which mean that the relations $a(x, t) \lesssim b(x, t)$ and $a(x, t) \gtrsim b(x, t)$, respectively, hold uniformly for $t \in \Delta$.

We are now ready to state the main result of this paper:

Theorem 2.1. Consider the insurance risk model introduced in Section 1 in which the claimsize distribution $F$ belongs to the class $\mathcal{R}_{-\alpha}$ for some $0<\alpha<\infty$ and the Laplace exponent of the Lévy process $\left\{R_{t}, t \geq 0\right\}$ satisfies $\phi\left(\alpha^{*}\right)<0$ for some $\alpha^{*}>\alpha$. Then, it holds uniformly for all $t \in \Lambda$ that

$$
\operatorname{Pr}\left(D_{t}>x\right) \sim \bar{F}(x) \int_{0-}^{t} \mathrm{e}^{s \phi(\alpha)} \mathrm{d} \lambda_{s} .
$$

Taking $t=\infty$ in relation (2.4) yields a more transparent asymptotic formula that

$$
\operatorname{Pr}\left(D_{\infty}>x\right) \sim \bar{F}(x) \frac{\mathrm{Ee}^{\phi(\alpha) \tau}}{1-\mathrm{Ee}^{\phi(\alpha) \tau}} .
$$

Roughly speaking, the condition $\phi\left(\alpha^{*}\right)<0$ in Theorem 2.1 means that the impact of the insurance claims dominates that of the financial uncertainty. This is also confirmed by relation (2.4), which shows that the tail probability of the claim-size distribution determines the exact decay rate while the financial uncertainty and the claim frequency only contribute to the coefficient of the asymptotic formula.

From (2.1) it is easy to verify that $\phi(z)$ is convex in $z$ for which $\phi(z)$ is finite. Since $\phi(0)=0$, we see that the condition $\phi\left(\alpha^{*}\right)<0$ implies that $\phi(z)<0$ for all $z \in\left(0, \alpha^{*}\right]$. In addition, by Jensen's inequality, the condition $\phi\left(\alpha^{*}\right)<0$ implies $\mathrm{E} R_{t}>0$. Hence, $R_{t}$ drifts to $\infty$ almost surely as $t \rightarrow \infty$.

As shown in Lemma 4.2 below, relation (2.4) with fixed $t \in \Lambda$ is an easy consequence of the one-dimensional version of Theorem 2.1 given in Resnick and Willekens (1991). However, it is much harder to prove the claimed uniformity of relation (2.4), which is in essence the scientific value of the present work.

Note that the result of Resnick and Willekens (1991) has recently been extended in many ways by Goovaerts et al. (2005), Wang and Tang (2006), Zhang et al. (2009), and Chen and Yuen (2009). Therefore, starting with these extended results, it should be possible and routine, but rather laborious, to further extend Theorem 2.1 to a somewhat broader class of heavy-tailed distributions (for example, the class of distributions with extended-regularlyvarying tails), and to the case that claim sizes possess a certain dependence structure (for example, pairwise asymptotic independence). We shall not pursue such extensions in this paper. However, it would be interesting to establish results similar to Theorem 2.1 in the presence of certain dependence structures among the sources of randomness, $\left\{X_{1}, X_{2}, \ldots\right\}$, $\left\{N_{t}, t \geq 0\right\}$, and $\left\{R_{t}, t \geq 0\right\}$. 


\section{Applications to Ruin Theory}

\subsection{Finite- and Infinite-time Ruin Probabilities}

Consider an insurance business commencing at time 0 with initial wealth $x \geq 0$. The cash flow of premiums less claims is modeled as a compound renewal process with the form

$$
C_{t}=c t-S_{t}, \quad t \geq 0,
$$

where $c \geq 0$ is a fixed rate of premium payment and $\left\{S_{t}, t \geq 0\right\}$ is a compound renewal process given in (1.1). Recall that the price process of the investment portfolio is the geometric Lévy process $\left\{\mathrm{e}^{R_{t}}, t \geq 0\right\}$. Thus, the wealth process of the insurer is described as

$$
U_{t}=\mathrm{e}^{R_{t}}\left(x+\int_{0-}^{t} \mathrm{e}^{-R_{s-}} \mathrm{d} C_{s}\right), \quad t \geq 0 .
$$

As usual, define the ruin time of this risk model as

$$
T(x)=\inf \left\{t>0: U_{t}<0 \mid U_{0}=x\right\}
$$

with the convention that $\inf \varnothing=\infty$. Then, the probability of ruin by a finite time $t \geq 0$ is

$$
\psi(x, t)=\operatorname{Pr}(T(x) \leq t),
$$

and the probability of ultimate ruin is

$$
\psi(x, \infty)=\operatorname{Pr}(T(x)<\infty)
$$

Yuen et al. (2004, 2006) studied the infinite-time ruin probability and related quantities of this renewal risk model. For the special case that $\left\{N_{t}, t \geq 0\right\}$ is a Poisson process, following their approach, it is not hard to establish an integro-differential equation and an integral equation for the infinite-time ruin probability. We shall not extend such a discussion here as we are mainly interested in the asymptotic behavior of the finite- and infinite-time ruin probabilities.

Theorem 3.1. Consider the insurance risk model introduced above. Under the conditions of Theorem 2.1, it holds uniformly for all $t \in \Lambda$ that

$$
\psi(x, t) \sim \bar{F}(x) \int_{0-}^{t} \mathrm{e}^{s \phi(\alpha)} \mathrm{d} \lambda_{s} .
$$

In particular, putting $t=\infty$ gives

$$
\psi(x, \infty) \sim \bar{F}(x) \frac{\mathrm{Ee}^{\phi(\alpha) \tau}}{1-\mathrm{Ee}^{\phi(\alpha) \tau}} .
$$

Paulsen (2002) obtained relation (3.4) for the special case that $\left\{N_{t}, t \geq 0\right\}$ is a Poisson process and $\left\{R_{t}, t \geq 0\right\}$ is a Brownian motion with positive drift; see Proposition 4.1 of 
Paulsen (2002). The reader is also referred to Heyde and Wang (2009) for a result for the finite-time ruin probability similar to (3.3) but with a fixed-time horizon and $\left\{N_{t}, t \geq 0\right\}$ being a Poisson process.

Due to the uniformity of relation (3.3), we can easily derive an explicit asymptotic expression for the Laplace transform of the ruin time $T(x)$.

Corollary 3.1. Under the conditions of Theorem 2.1, it holds for every $r \geq 0$ that

$$
\mathrm{Ee}^{-r T(x)} \sim \bar{F}(x) \frac{\mathrm{Ee}^{(\phi(\alpha)-r) \tau}}{1-\mathrm{Ee}^{(\phi(\alpha)-r) \tau}} .
$$

Proof. When $r=0$, relation (3.5) coincides with relation (3.4). Thus, we only need to consider $r>0$. By the uniformity of relation (3.3), we have

$$
\mathrm{Ee}^{-r T(x)}=r \int_{0}^{\infty} \psi(x, t) \mathrm{e}^{-r t} \mathrm{~d} t \sim r \bar{F}(x) \int_{0}^{\infty}\left[\int_{0-}^{t} \mathrm{e}^{\phi(\alpha) s} \mathrm{~d} \lambda_{s}\right] \mathrm{e}^{-r t} \mathrm{~d} t .
$$

Using Fubini's theorem to interchange the order of integrals,

$$
\int_{0}^{\infty}\left[\int_{0-}^{t} \mathrm{e}^{\phi(\alpha) s} \mathrm{~d} \lambda_{s}\right] \mathrm{e}^{-r t} \mathrm{~d} t=\int_{0-}^{\infty}\left[\int_{s}^{\infty} \mathrm{e}^{-r t} \mathrm{~d} t\right] \mathrm{e}^{\phi(\alpha) s} \mathrm{~d} \lambda_{s}=\frac{1}{r} \int_{0-}^{\infty} \mathrm{e}^{(\phi(\alpha)-r) s} \mathrm{~d} \lambda_{s} .
$$

Therefore, (3.5) holds.

\subsection{Portfolio Optimization with a Constraint on Ruin}

It is commonly acknowledged that risky investments may impair the insurer's solvency just as severely as large claims do; see Kalashnikov and Norberg (2002) and Tang and Tsitsiashvili (2003). Frolova et al. (2002) also pointed out that disasters may arrive at the period when the market value of assets is low and the company will not be able to cover losses by selling these assets.

In this section, we consider a so-called constant investment portfolio and determine the optimal investment strategy that maximizes the insurer's expected terminal wealth and maintains the insurer's solvency. Such optimization problems have been considered by several authors; see, for example, Schmidli (2002), Paulsen (2003), and Kostadinova (2007).

For simplicity, we assume that a financial market consists of two assets, which can be traded continuously. One of them is a risk-free asset with price process satisfying

$$
\mathrm{d} P_{t}^{(0)}=r_{0} P_{t}^{(0)} \mathrm{d} t, \quad t>0,
$$

with $P_{0}^{(0)}=1$ and $r_{0}>0$, while the other is a risky asset with price process satisfying

$$
\mathrm{d} P_{t}^{(1)}=P_{t-}^{(1)} \mathrm{d} Q_{t}, \quad t>0,
$$

with $P_{0}^{(1)}=1$ and $\left\{Q_{t}, t \geq 0\right\}$ being a Lévy process with Lévy triplet $\left(r_{Q}, \sigma_{Q}^{2}, \rho_{Q}\right)$, as described in Section 2. Suppose that the insurer continuously invests a constant fraction 
$\pi \in[0,1]$ of his wealth in the risky asset and keeps the remaining wealth in the risk-free asset. This constant investment strategy is commonly used in mathematical finance and actuarial science; see, for example, Emmer et al. (2001), Björk (1998), Emmer and Klüppelberg (2004), Kostadinova (2007), and Klüppelberg and Kostadinova (2008).

The price process of this investment portfolio satisfies the stochastic differential equation

$$
\mathrm{d} P_{t}^{(\pi)}=P_{t-}^{(\pi)} \mathrm{d} Q_{t}^{(\pi)}, \quad t>0,
$$

with $P_{0}^{(\pi)}=1$ and $Q_{t}^{(\pi)}=(1-\pi) r_{0} t+\pi Q_{t}$ for $t \geq 0$. From Theorem 4.1 of Cont and Tankov (2004) (see also Proposition 11.10 of Sato (1999)), the Lévy triplet of the Lévy process $\left\{Q_{t}^{(\pi)}, t \geq 0\right\}$ is given by

$$
\left\{\begin{array}{l}
r_{\pi}=(1-\pi) r_{0}+\pi r_{Q}+\int_{-\infty}^{\infty} y\left(1_{(-1,1)}(y)-1_{\Theta}(y)\right) \rho_{\pi}(\mathrm{d} y), \\
\sigma_{\pi}^{2}=\pi^{2} \sigma_{Q}^{2} \\
\rho_{\pi}(A)=\rho_{Q}(\{x: \pi x \in A\}) \text { for every Borel set } A
\end{array}\right.
$$

where $\Theta=\{\pi x:|x|<1\}$. Hence, by Proposition 8.22 of Cont and Tankov (2004) or Theorem 37 in Chapter 2 of Protter (2005), we solve (3.7) to get

$$
P_{t}^{(\pi)}=\mathrm{e}^{R_{t}^{(\pi)}}, \quad t \geq 0
$$

where

$$
R_{t}^{(\pi)}=(1-\pi) r_{0} t+\pi Q_{t}-\frac{1}{2} \sigma_{Q}^{2} \pi^{2} t+\sum_{0<s \leq t}\left(\ln \left(1+\pi \Delta Q_{s}\right)-\pi \Delta Q_{s}\right),
$$

with $\Delta Q_{s}=Q_{s}-Q_{s-}$. The solution $P_{t}^{(\pi)}$ given above is recognized as the stochastic exponential of the Lévy process $\left\{Q_{t}^{(\pi)}, t \geq 0\right\}$. By Proposition 8.22 of Cont and Tankov (2004), the Lévy triplet of $\left\{R_{t}^{(\pi)}, t \geq 0\right\}$ is given by

$$
\left\{\begin{array}{l}
r=r_{\pi}-\frac{1}{2} \sigma_{\pi}^{2}+\int_{-\infty}^{\infty}\left(\ln (1+y) 1_{(-1,1)}(\ln (1+y))-y 1_{(-1,1)}(y)\right) \rho_{\pi}(\mathrm{d} y) \\
\sigma^{2}=\sigma_{\pi}^{2} \\
\rho(A)=\rho_{\pi}(\{y: \ln (1+y) \in A\}) \text { for every Borel set } A
\end{array}\right.
$$

Using the Lévy triplet of $\left\{Q_{t}, t \geq 0\right\}$, one obtains the Laplace exponent of $\left\{R_{t}^{(\pi)}, t \geq 0\right\}$ as

$$
\begin{aligned}
\phi_{\pi}(z)= & \frac{1}{2} \pi^{2} \sigma_{Q}^{2} z^{2}-\left((1-\pi) r_{0}+\pi r_{Q}-\frac{1}{2} \pi^{2} \sigma_{Q}^{2}-\pi \int_{-1}^{1} y \rho_{Q}(\mathrm{~d} y)\right) z \\
& +\int_{-\infty}^{\infty}\left((1+\pi y)^{-z}-1\right) \rho_{Q}(\mathrm{~d} y)
\end{aligned}
$$

provided that the second integral in $(3.8)$ is finite. Note that $\rho_{Q}((-\infty,-1])=0$ since the price process $\left\{P_{t}^{(1)}, t \geq 0\right\}$ in (3.6) is positive. It follows from (3.8) that

$$
\phi_{\pi}(-1)=r_{0}+\pi \xi
$$

where $\xi=r_{Q}-r_{0}+\int_{1}^{\infty} y \rho_{Q}(\mathrm{~d} y)$. 
Consider a fixed-time horizon $t_{0}>0$, say $t_{0}=5$. Our goal is to determine a value $\pi_{*} \in[0,1]$ that maximizes the expected value of the terminal wealth

$$
U_{t_{0}}^{(\pi)}=\mathrm{e}^{R_{t_{0}}^{(\pi)}}\left(x+\int_{0-}^{t_{0}} \mathrm{e}^{-R_{s-}^{(\pi)}} \mathrm{d} C_{s}\right)
$$

subject to the following constraint on the ruin probability:

$$
\psi_{\pi}\left(x, t_{0}\right)=\operatorname{Pr}\left(\inf _{0 \leq s \leq t_{0}} U_{s}^{(\pi)}<0 \mid U_{0}=x\right) \leq p,
$$

where $\left\{C_{t}, t \geq 0\right\}$ is given in (3.1) and $p$ is some small positive number, say $p=5 \%$.

Let the conditions of Theorem 3.1 hold, and let $\alpha>1$ so that the i.i.d. claims have a finite mean $\mu=\mathrm{E} X$. Simple calculation gives

$$
\mathrm{E} U_{t_{0}}^{(\pi)}=x \mathrm{e}^{\phi_{\pi}(-1) t_{0}}+\int_{0-}^{t_{0}} \mathrm{e}^{\phi_{\pi}(-1)\left(t_{0}-s\right)} \mathrm{d}\left(c s-\mu \lambda_{s}\right) .
$$

Since there is usually no closed-form expression for $\psi_{\pi}\left(x, t_{0}\right)$ available, we use its approximation given in (2.4); that is to say, we replace (3.11) by

$$
\psi_{\pi}\left(x, t_{0}\right) \sim \bar{F}(x) \int_{0-}^{t_{0}} \mathrm{e}^{\phi_{\pi}(\alpha) s} \mathrm{~d} \lambda_{s} \leq p .
$$

For given $x>0, \alpha>1, t_{0}>0$, and $0<p<1$, define

$$
\Pi=\left\{\pi: \pi \in[0,1] \text { and } \bar{F}(x) \int_{0-}^{t_{0}} \mathrm{e}^{\phi_{\pi}(\alpha) s} \mathrm{~d} \lambda_{s} \leq p\right\} .
$$

Suppose $\varnothing \neq \Pi \subset\left\{\pi: \phi_{\pi}(\alpha)<0\right\}$. Every $\pi \in \Pi$ is called an admissible investment strategy. With $\alpha>1$ given, we see from (3.8) that $\phi_{\pi}(\alpha)$, as a function of $\pi$, is convex in $\pi \in[0,1]$ with $\phi_{0}(\alpha)=-r_{0} \alpha<0$. Hence, $\Pi$ is a closed interval in $[0,1]$. Let $\Pi=\left[a_{x}, b_{x}\right] \subset[0,1]$.

To simplify the optimization problem, we assume that $\lambda_{s}$ is absolutely continuous with respect to the Lebesgue measure; that is to say, there exists a nonnegative and measurable function $\lambda_{s}^{\prime}$ such that $\lambda_{s}-\lambda_{0}=\int_{0}^{s} \lambda_{u}^{\prime} \mathrm{d} u$ for $s \in[0, \infty)$. We further assume that the relation

$$
c-\mu \lambda_{s}^{\prime} \geq 0
$$

holds almost everywhere for $s \in[0, \infty)$. Relation (3.14) can be interpreted as the safety loading condition of the insurance portfolio. It is verifiable for many interesting cases, for example, when $\tau$ follows an exponential distribution or a $\Gamma(2, \lambda)$ distribution.

Under (3.14), the expectation $\mathrm{E} U_{t_{0}}^{(\pi)}$, as expressed in $(3.12)$, is increasing in $\phi_{\pi}(-1)$. Hence, the optimization problem becomes

$$
\text { maximizing } \phi_{\pi}(-1) \quad \text { subject to } \pi \in \Pi \text {. }
$$

From (3.9), we see that $\phi_{\pi}(-1)$ is increasing in $\pi \in[0,1]$ when $\xi>0$ and decreasing in $\pi \in[0,1]$ when $\xi<0$. Hence, if $\xi>0$, the solution to $(3.15)$ is

$$
\pi_{*}=b_{x},
$$


while if $\xi<0$, the solution to $(3.15)$ is

$$
\pi_{*}=a_{x}
$$

When $\xi=0$, however, $\phi_{\pi}(-1)=r_{0}$ does not depend on $\pi$. Thus, every admissible strategy $\pi \in \Pi$ could be used as a solution to (3.15). Nevertheless, in order to reduce uncertainty from the risky asset, we may choose (3.17) as the solution.

The optimization solutions (3.16) and (3.17) are intuitively clear. The condition $\xi>0$ means that the expected return rate of the risky asset is higher than that of the risk-free asset. Hence, as (3.16) shows, the insurer will invest as much as he is allowed in the risky asset. The optimization solution (3.17) can be explained in a similar way. Moreover, we have the following observation. In modern portfolio theory, the quantity $\sigma_{Q}^{2}$, which is the volatility when $\left\{Q_{t}, t \geq 0\right\}$ is a Brownian motion with drift, is often used to measure the risk of the risky asset. Recall relation (3.8) and the definition of $\Pi$. Clearly, $\phi_{\pi}(\alpha)$ is increasing in $\sigma_{Q}^{2}$. Hence, both $a_{x}$ and $b_{x}$ are decreasing in $\sigma_{Q}^{2}$, meaning that the riskier the risky asset is, the less the insurer will invest in it.

\section{Proofs}

\subsection{Lemmas}

Let us first recall some properties of distributions with regularly-varying tails. By Theorem 1.5.2 of Bingham et al. (1987), the convergence in relation $(2.3)$ is uniform over $[\varepsilon, \infty)$ for every fixed $\varepsilon>0$; that is to say,

$$
\lim _{x \rightarrow \infty} \sup _{y \in[\varepsilon, \infty)}\left|\frac{\bar{F}(x y)}{\bar{F}(x)}-y^{-\alpha}\right|=0 .
$$

By Theorem 1.5.6 of Bingham et al. (1987), the well-known Potter's bounds for distributions in the class $\mathcal{R}$ are stated as follows. If $F \in \mathcal{R}_{-\alpha}$ for some $0<\alpha<\infty$, then for arbitrarily fixed $b>1$ and $\varepsilon>0$, there exists some $x_{0}>0$ such that, for all $x, y \geq x_{0}$,

$$
\frac{1}{b}\left((y / x)^{-\alpha+\varepsilon} \wedge(y / x)^{-\alpha-\varepsilon}\right) \leq \frac{\bar{F}(y)}{\bar{F}(x)} \leq b\left((y / x)^{-\alpha+\varepsilon} \vee(y / x)^{-\alpha-\varepsilon}\right) .
$$

For arbitrarily fixed $0<\alpha_{*}<\alpha<\alpha^{*}<\infty$, by fixing the variable $y$ to $x_{0}$ and $\varepsilon<$ $\left(\alpha-\alpha_{*}\right) \wedge\left(\alpha^{*}-\alpha\right)$ in $(4.2)$, we see that

$$
\bar{F}(x)=o\left(x^{-\alpha_{*}}\right), \quad x^{-\alpha^{*}}=o(\bar{F}(x)) .
$$

Let $X$ and $Y$ be two independent random variables with $X$ following a distribution $F \in \mathcal{R}_{-\alpha}$ for some $0<\alpha<\infty$ and $Y$ being a nonnegative random variable satisfying $\mathrm{E}^{\alpha^{*}}<\infty$ for some $\alpha^{*}>\alpha$. Then, for every fixed $M \geq 0$, with $x_{0}>0$ given in (4.2), we have

$$
\begin{aligned}
\lim _{x \rightarrow \infty} \frac{\operatorname{Pr}(X Y>x, Y>M)}{\bar{F}(x)} & =\lim _{x \rightarrow \infty} \frac{\operatorname{Pr}\left(X Y>x, Y \in\left(M, x / x_{0}\right] \cup\left(x / x_{0}, \infty\right)\right)}{\bar{F}(x)} \\
& =\mathrm{E}^{\alpha} 1_{(Y>M)},
\end{aligned}
$$


where we used the dominated convergence theorem guaranteed by (4.2) in dealing with the first part corresponding to $Y \in\left(M, x / x_{0}\right]$, and used Markov's inequality and the second relation of (4.3) in dealing with the second part. Relation (4.4) with $M=0$ is well known, usually referred to as Breiman's theorem; see Breiman (1965).

The following result is the one-dimensional version of Theorem 2.1 of Resnick and Willekens (1991):

Lemma 4.1. Consider the randomly weighted sum

$$
S^{(W)}=\sum_{k=1}^{\infty} W_{k} X_{k}
$$

where $\left\{X_{1}, X_{2}, \ldots\right\}$ is a sequence of i.i.d. nonnegative random variables with common distribution $F \in \mathcal{R}_{-\alpha}$ for some $0<\alpha<\infty$, and $\left\{W_{1}, W_{2}, \ldots\right\}$ is another sequence of nonnegative random variables independent of $\left\{X_{1}, X_{2}, \ldots\right\}$. We have

$$
\operatorname{Pr}\left(S^{(W)}>x\right) \sim \bar{F}(x) \sum_{k=1}^{\infty} \mathrm{E} W_{k}^{\alpha},
$$

if one of the following assumptions holds:

1. $0<\alpha<1$ and for some $0<\varepsilon<\alpha \wedge(1-\alpha)$,

$$
\sum_{k=1}^{\infty} \mathrm{E}\left(W_{k}^{\alpha+\varepsilon} \vee W_{k}^{\alpha-\varepsilon}\right)<\infty
$$

2. $1 \leq \alpha<\infty$ and for some $0<\varepsilon<\alpha$,

$$
\sum_{k=1}^{\infty}\left(\mathrm{E}\left(W_{k}^{\alpha+\varepsilon} \vee W_{k}^{\alpha-\varepsilon}\right)\right)^{\frac{1}{\alpha+\varepsilon}}<\infty .
$$

Lemma 4.2. Under the conditions of Theorem 2.1, relation (2.4) holds for every fixed $t \in \Lambda$.

Proof. We apply Lemma 4.1 to the series $D_{t}$ given in (1.2). For $0<\alpha<1$, we choose some $0<\varepsilon<\alpha \wedge(1-\alpha) \wedge\left(\alpha^{*}-\alpha\right)$ so that

$$
\begin{aligned}
& \sum_{k=1}^{\infty} \mathrm{E}\left(\mathrm{e}^{-(\alpha+\varepsilon) R_{\tau_{k}}} \vee \mathrm{e}^{-(\alpha-\varepsilon) R_{\tau_{k}}}\right) 1_{\left(\tau_{k} \leq t\right)} \\
\leq & \sum_{k=1}^{\infty} \int_{0-}^{\infty} \mathrm{E}\left(\mathrm{e}^{-(\alpha+\varepsilon) R_{s}}+\mathrm{e}^{-(\alpha-\varepsilon) R_{s}}\right) \operatorname{Pr}\left(\tau_{k} \in \mathrm{d} s\right) \\
= & \frac{\mathrm{Ee}^{\phi(\alpha+\varepsilon) \tau}}{1-\mathrm{Ee}^{\phi(\alpha+\varepsilon) \tau}}+\frac{\mathrm{Ee}^{\phi(\alpha-\varepsilon) \tau}}{1-\mathrm{Ee}^{\phi(\alpha-\varepsilon) \tau}} \\
< & \infty
\end{aligned}
$$

Similarly, for $1 \leq \alpha<\infty$, we choose some $0<\varepsilon<\alpha \wedge\left(\alpha^{*}-\alpha\right)$ to justify that

$$
\sum_{k=1}^{\infty}\left(\mathrm{E}\left(\mathrm{e}^{-(\alpha+\varepsilon) R_{\tau_{k}}} \vee \mathrm{e}^{-(\alpha-\varepsilon) R_{\tau_{k}}}\right)\right)^{\frac{1}{\alpha+\varepsilon}} 1_{\left(\tau_{k} \leq t\right)}<\infty .
$$


Therefore, by Lemma 4.1,

$$
\operatorname{Pr}\left(D_{t}>x\right) \sim \bar{F}(x) \sum_{k=1}^{\infty} \mathrm{Ee}^{-\alpha R_{\tau_{k}}} 1_{\left(\tau_{k} \leq t\right)}=\bar{F}(x) \int_{0-}^{t} \mathrm{e}^{s \phi(\alpha)} \mathrm{d} \lambda_{s} .
$$

This proves that relation (2.4) holds for every fixed $t \in \Lambda$.

Lemma 4.3. Under the conditions of Theorem 2.1, for every fixed $T \in \Lambda$, it holds uniformly for all $t \in \Lambda_{T}$ that

$$
\operatorname{Pr}\left(X \mathrm{e}^{-R_{\tau}} 1_{(\tau \leq t)}>x\right) \sim \bar{F}(x) \int_{0-}^{t} \mathrm{e}^{s \phi(\alpha)} \operatorname{Pr}(\tau \in \mathrm{d} s) .
$$

Proof. Conditioning on $\tau$, we have

$$
\operatorname{Pr}\left(X \mathrm{e}^{-R_{\tau}} 1_{(\tau \leq t)}>x\right)=\int_{0-}^{t} \operatorname{Pr}\left(X \mathrm{e}^{-R_{s}}>x\right) \operatorname{Pr}(\tau \in \mathrm{d} s) .
$$

For an arbitrarily fixed large number $M>0$, according to $\left(\left|R_{s}\right| \leq M\right),\left(R_{s}>M\right)$, and $\left(R_{s}<-M\right)$, we split the right-hand side of the above into three parts as $I_{1}(x, t)+I_{2}(x, t)+$ $I_{3}(x, t)$. By relation (4.1), it holds uniformly for all $t \in \Lambda$ that

$$
\begin{aligned}
I_{1}(x, t) & =\int_{0-}^{t} \operatorname{Pr}\left(X \mathrm{e}^{-R_{s}}>x,\left|R_{s}\right| \leq M\right) \operatorname{Pr}(\tau \in \mathrm{d} s) \\
& \sim \bar{F}(x) \int_{0-}^{t} \operatorname{Ee}^{-\alpha R_{s}} 1_{\left(\left|R_{s}\right| \leq M\right)} \operatorname{Pr}(\tau \in \mathrm{d} s) \\
& =\bar{F}(x) \int_{0-}^{t} \mathrm{e}^{s \phi(\alpha)} \operatorname{Pr}(\tau \in \mathrm{d} s)-\bar{F}(x) \int_{0-}^{t} \mathrm{Ee}^{-\alpha R_{s}} 1_{\left(\left|R_{s}\right|>M\right)} \operatorname{Pr}(\tau \in \mathrm{d} s) .
\end{aligned}
$$

Thus, it suffices to show that the terms $I_{2}(x, t), I_{3}(x, t)$, and the last term above, denoted as $I_{4}(x, t)$, are negligible for large $M>0$, uniformly for all $t \in \Lambda_{T}$, in comparison to $\bar{F}(x) \int_{0-}^{t} \mathrm{e}^{s \phi(\alpha)} \operatorname{Pr}(\tau \in \mathrm{d} s)$. More precisely, we are going to prove that, for $j=2,3,4$,

$$
\limsup _{M \rightarrow \infty} \limsup _{x \rightarrow \infty} \sup _{t \in \Lambda_{T}} \frac{I_{j}(x, t)}{\bar{F}(x) \int_{0-}^{t} \mathrm{e}^{s \phi(\alpha)} \operatorname{Pr}(\tau \in \mathrm{d} s)}=0 .
$$

For $I_{2}(x, t)$, we have

$$
\begin{aligned}
I_{2}(x, t) & =\int_{0-}^{t} \operatorname{Pr}\left(X \mathrm{e}^{-R_{s}}>x, R_{s}>M\right) \operatorname{Pr}(\tau \in \mathrm{d} s) \\
& \leq \operatorname{Pr}\left(X \mathrm{e}^{-M}>x\right) \operatorname{Pr}(\tau \leq t) \\
& \sim \mathrm{e}^{-\alpha M} \bar{F}(x) \operatorname{Pr}(\tau \leq t) .
\end{aligned}
$$

Trivially,

$$
\operatorname{Pr}(\tau \leq t) \leq \mathrm{e}^{-T \phi(\alpha)} \int_{0-}^{t} \mathrm{e}^{s \phi(\alpha)} \operatorname{Pr}(\tau \in \mathrm{d} s)
$$


This proves relation (4.6) with $j=2$. For $I_{3}(x, t)$, we have

$$
\begin{aligned}
I_{3}(x, t) & =\int_{0-}^{t} \operatorname{Pr}\left(X \mathrm{e}^{-R_{s}}>x, R_{s}<-M\right) \operatorname{Pr}(\tau \in \mathrm{d} s) \\
& \leq \operatorname{Pr}\left(X \mathrm{e}^{-\inf _{0 \leq s \leq T} R_{s}}>x, \inf _{0 \leq s \leq T} R_{s}<-M\right) \operatorname{Pr}(\tau \leq t) .
\end{aligned}
$$

Note that, for all $T \geq 0$ and $x>x_{0}>0$,

$$
\operatorname{Pr}\left(-\inf _{0 \leq s \leq T} R_{s}>x\right) \operatorname{Pr}\left(-\sup _{0 \leq s \leq T} R_{s}>-x_{0}\right) \leq \operatorname{Pr}\left(-R_{T}>x-x_{0}\right) ;
$$

see the lemma of Willekens (1987). This, together with $\mathrm{Ee}^{-\alpha^{*} R_{T}}=\mathrm{e}^{T \phi\left(\alpha^{*}\right)}<1$, imply that

$$
\mathrm{Ee}^{-\alpha^{*} \inf _{0 \leq s \leq T} R_{s}}<\infty .
$$

Hence, applying (4.4) to (4.8), it holds uniformly for all $t \in \Lambda_{T}$ that

$$
I_{3}(x, t) \lesssim \mathrm{Ee}^{-\alpha \inf _{0 \leq s \leq T} R_{s}} 1_{\left(\inf _{0 \leq s \leq T} R_{s}<-M\right)} \bar{F}(x) \operatorname{Pr}(\tau \leq t) .
$$

This, together with (4.7), prove relation (4.6) with $j=3$. Finally, for $I_{4}(x, t)$, by Hölder's inequality,

$$
\begin{aligned}
& \int_{0-}^{t} \operatorname{Ee}^{-\alpha R_{s}} 1_{\left(\left|R_{s}\right|>M\right)} \operatorname{Pr}(\tau \in \mathrm{d} s) \\
\leq & \int_{0-}^{t}\left(\mathrm{Ee}^{-\alpha^{*} R_{s}}\right)^{\alpha / \alpha^{*}} \operatorname{Pr}\left(\left|R_{s}\right|>M\right)^{1-\alpha / \alpha^{*}} \operatorname{Pr}(\tau \in \mathrm{d} s) \\
\leq & \operatorname{Pr}\left(\sup _{0 \leq s \leq T}\left|R_{s}\right|>M\right)^{1-\alpha / \alpha^{*}} \operatorname{Pr}(\tau \leq t) .
\end{aligned}
$$

Hence, by (4.7), relation (4.6) with $j=4$ holds and we conclude the proof.

Lemma 4.4. Under the conditions of Theorem 2.1, for every fixed $T \in \Lambda$ and $n=1,2, \ldots$, it holds uniformly for all $t \in \Lambda_{T}$ that

$$
\operatorname{Pr}\left(\sum_{k=1}^{n} X_{k} \mathrm{e}^{-R_{\tau_{k}}} 1_{\left(\tau_{k} \leq t\right)}>x\right) \sim \bar{F}(x) \int_{0-}^{t} \mathrm{e}^{s \phi(\alpha)} \sum_{k=1}^{n} \operatorname{Pr}\left(\tau_{k} \in \mathrm{d} s\right) .
$$

Proof. By Lemma 4.3, it suffices to prove that, uniformly for all $t \in \Lambda_{T}$,

$$
\operatorname{Pr}\left(\sum_{k=1}^{n} X_{k} \mathrm{e}^{-R_{\tau_{k}}} 1_{\left(\tau_{k} \leq t\right)}>x\right) \sim \sum_{k=1}^{n} \operatorname{Pr}\left(X_{k} \mathrm{e}^{-R_{\tau_{k}}} 1_{\left(\tau_{k} \leq t\right)}>x\right) .
$$

We first prove the lower-bound version of relation (4.9). Clearly,

$$
\begin{aligned}
& \operatorname{Pr}\left(\sum_{k=1}^{n} X_{k} \mathrm{e}^{-R_{\tau_{k}}} 1_{\left(\tau_{k} \leq t\right)}>x\right) \\
\geq & \operatorname{Pr}\left(\bigcup_{k=1}^{n} X_{k} \mathrm{e}^{-R_{\tau_{k}}} 1_{\left(\tau_{k} \leq t\right)}>x\right) \\
\geq & \sum_{k=1}^{n} \operatorname{Pr}\left(X_{k} \mathrm{e}^{-R_{\tau_{k}}} 1_{\left(\tau_{k} \leq t\right)}>x\right)-\sum_{1 \leq k<j \leq n} \operatorname{Pr}\left(X_{k} \mathrm{e}^{\left.-R_{\tau_{k}} 1_{\left(\tau_{k} \leq t\right)}>x, X_{j} \mathrm{e}^{-R_{\tau_{j}}} 1_{\left(\tau_{j} \leq t\right)}>x\right) .}\right.
\end{aligned}
$$


Thus, it remains to verify that the second term above is negligible, uniformly for all $t \in \Lambda_{T}$, in comparison to the first term. Actually, for arbitrarily fixed $1 \leq k<j \leq n$ and $M>0$,

$$
\begin{aligned}
& \operatorname{Pr}\left(X_{k} \mathrm{e}^{-R_{\tau_{k}}} 1_{\left(\tau_{k} \leq t\right)}>x, X_{j} \mathrm{e}^{-R_{\tau_{j}}} 1_{\left(\tau_{j} \leq t\right)}>x\right) \\
& \leq \int_{0-}^{t} \operatorname{Pr}\left(X_{k} \mathrm{e}^{-R_{s}}>x, \mathrm{e}^{-R_{s}} X_{j} \mathrm{e}^{-R_{\tau_{j}}+R_{\tau_{k}}} 1_{\left(\tau_{j} \leq t\right)}>x, \mathrm{e}^{-R_{s}} \leq \frac{x}{M} \mid \tau_{k}=s\right) \operatorname{Pr}\left(\tau_{k} \in \mathrm{d} s\right) \\
& +\int_{0-}^{t} \operatorname{Pr}\left(\mathrm{e}^{-R_{s}}>\frac{x}{M}\right) \operatorname{Pr}\left(\tau_{k} \in \mathrm{d} s\right) \\
& =I_{1}(x, t)+I_{2}(x, t) \text {. }
\end{aligned}
$$

Note that $\mathrm{e}^{-R_{s}}$ and $\mathrm{e}^{-R_{\tau_{j}}+R_{\tau_{k}}}$ in $I_{1}(x, t)$ conditional on $\left(\tau_{k}=s\right)$ are independent. It holds for arbitrarily fixed $\varepsilon>0$ and all large $M>0$ that

$$
\begin{aligned}
I_{1}(x, t) & \leq \operatorname{Pr}\left(X_{j} \sup _{0 \leq s \leq T} \mathrm{e}^{-R_{s}}>M\right) \int_{0-}^{t} \operatorname{Pr}\left(X_{k} \mathrm{e}^{-R_{s}}>x\right) \operatorname{Pr}\left(\tau_{k} \in \mathrm{d} s\right) \\
& \leq \varepsilon \operatorname{Pr}\left(X_{k} \mathrm{e}^{-R_{\tau_{k}}} 1_{\left(\tau_{k} \leq t\right)}>x\right) .
\end{aligned}
$$

For $I_{2}(x, t)$, by Markov's inequality, the second relation of (4.3), relation (4.7), and Lemma 4.3 , we have

$$
\begin{aligned}
I_{2}(x, t) & \leq \int_{0-}^{t}\left(\frac{x}{M}\right)^{-\alpha^{*}} \mathrm{Ee}^{-\alpha^{*} R_{s}} \operatorname{Pr}\left(\tau_{k} \in \mathrm{d} s\right) \\
& \leq\left(\frac{x}{M}\right)^{-\alpha^{*}} \operatorname{Pr}\left(\tau_{k} \leq t\right) \\
& =o(1) \bar{F}(x) \int_{0-}^{t} \mathrm{e}^{s \phi(\alpha)} \operatorname{Pr}\left(\tau_{k} \in \mathrm{d} s\right) \\
& =o(1) \operatorname{Pr}\left(X_{k} \mathrm{e}^{-R_{\tau_{k}}} 1_{\left(\tau_{k} \leq t\right)}>x\right) .
\end{aligned}
$$

Therefore, it holds uniformly for all $t \in \Lambda_{T}$ that

$$
\operatorname{Pr}\left(X_{k} \mathrm{e}^{-R_{\tau_{k}}} 1_{\left(\tau_{k} \leq t\right)}>x, X_{j} \mathrm{e}^{-R_{\tau_{j}}} 1_{\left(\tau_{j} \leq t\right)}>x\right)=o(1) \operatorname{Pr}\left(X_{k} \mathrm{e}^{-R_{\tau_{k}}} 1_{\left(\tau_{k} \leq t\right)}>x\right) .
$$

We next prove the upper-bound version of relation (4.9). For arbitrarily fixed $0<\delta<1$, we derive

$$
\begin{aligned}
& \operatorname{Pr}\left(\sum_{k=1}^{n} X_{k} \mathrm{e}^{-R_{\tau_{k}}} 1_{\left(\tau_{k} \leq t\right)}>x\right) \\
\leq & \operatorname{Pr}\left(\bigcup_{k=1}^{n}\left(X_{k} \mathrm{e}^{-R_{\tau_{k}}} 1_{\left(\tau_{k} \leq t\right)}>x-\delta x\right)\right) \\
& +\operatorname{Pr}\left(\sum_{k=1}^{n} X_{k} \mathrm{e}^{-R_{\tau_{k}}} 1_{\left(\tau_{k} \leq t\right)}>x, \bigcup_{k=1}^{n}\left(X_{k} \mathrm{e}^{-R_{\tau_{k}}} 1_{\left(\tau_{k} \leq t\right)}>\frac{x}{n}\right), \bigcap_{k=1}^{n}\left(X_{k} \mathrm{e}^{-R_{\tau_{k}}} 1_{\left(\tau_{k} \leq t\right)} \leq x-\delta x\right)\right) \\
= & I_{3}(x, t)+I_{4}(x, t) .
\end{aligned}
$$


By Lemma 4.3, it holds uniformly for all $t \in \Lambda_{T}$ that

$$
I_{3}(x, t) \leq \sum_{k=1}^{n} \operatorname{Pr}\left(X_{k} \mathrm{e}^{-R_{\tau_{k}}} 1_{\left(\tau_{k} \leq t\right)}>x-\delta x\right) \sim(1-\delta)^{-\alpha} \sum_{k=1}^{n} \operatorname{Pr}\left(X_{k} \mathrm{e}^{-R_{\tau_{k}}} 1_{\left(\tau_{k} \leq t\right)}>x\right) .
$$

Thus, it remains to verify that $I_{4}(x, t)$ is negligible, uniformly for all $t \in \Lambda_{T}$, in comparison to $I_{3}(x, t)$. Actually, it holds uniformly for all $t \in \Lambda_{T}$ that

$$
\begin{aligned}
& I_{4}(x, t) \leq \sum_{k=1}^{n} \operatorname{Pr}\left(X_{k} \mathrm{e}^{-R_{\tau_{k}}} 1_{\left(\tau_{k} \leq t\right)}>\frac{x}{n}, \sum_{j \in\{1, \ldots, n\} \backslash\{k\}} X_{j} \mathrm{e}^{\left.-R_{\tau_{j}} 1_{\left(\tau_{j} \leq t\right)}>\delta x\right)}\right. \\
& \leq \sum_{1 \leq k \neq j \leq n} \operatorname{Pr}\left(X_{k} \mathrm{e}^{-R_{\tau_{k}}} 1_{\left(\tau_{k} \leq t\right)}>\frac{\delta x}{n}, X_{j} \mathrm{e}^{-R_{\tau_{j}}} 1_{\left(\tau_{j} \leq t\right)}>\frac{\delta x}{n}\right) \\
& =o(1) \sum_{k=1}^{n} \operatorname{Pr}\left(X_{k} \mathrm{e}^{-R_{\tau_{k}}} 1_{\left(\tau_{k} \leq t\right)}>x\right) \text {, }
\end{aligned}
$$

where in the last step we used relation (4.10) and Lemma 4.3. This ends the proof.

Lemma 4.5. Under the conditions of Theorem 2.1, for every fixed $T \in \Lambda$, it holds for every $\varepsilon>0$, for all large $n$, and uniformly for all $t \in \Lambda_{T}$ that

$$
\operatorname{Pr}\left(\sum_{k=n+1}^{\infty} X_{k} \mathrm{e}^{-R_{\tau_{k}}} 1_{\left(\tau_{k} \leq t\right)}>x\right) \lesssim \varepsilon \bar{F}(x) \int_{0-}^{t} \mathrm{e}^{s \phi(\alpha)} \mathrm{d} \lambda_{s}
$$

Proof. Choose some $\delta>0$ such that $(1-\delta) \alpha^{*}>\alpha$. Then,

$$
\begin{aligned}
& \operatorname{Pr}\left(\sum_{k=n+1}^{\infty} X_{k} \mathrm{e}^{-R_{\tau_{k}}} 1_{\left(\tau_{k} \leq t\right)}>x\right) \\
\leq & \int_{0-}^{t} \operatorname{Pr}\left(\mathrm{e}^{-R_{s}} \sum_{k=n+1}^{\infty} X_{k} \mathrm{e}^{-R_{\tau_{k}}+R_{\tau_{n}}} 1_{\left(\tau_{k} \leq t\right)}>x, \mathrm{e}^{-R_{s}} \leq x^{1-\delta} \mid \tau_{n}=s\right) \operatorname{Pr}\left(\tau_{n} \in \mathrm{d} s\right) \\
& \quad+\int_{0-}^{t} \operatorname{Pr}\left(\mathrm{e}^{-R_{s}}>x^{1-\delta}\right) \operatorname{Pr}\left(\tau_{n} \in \mathrm{d} s\right) \\
= & I_{1}(x, t)+I_{2}(x, t) .
\end{aligned}
$$

For $I_{1}(x, t)$, note that, by Lemma 4.2 , it holds uniformly for all $0 \leq s \leq t \in \Lambda_{T}$ that

$$
\begin{aligned}
\operatorname{Pr}\left(\sum_{k=n+1}^{\infty} X_{k} \mathrm{e}^{-R_{\tau_{k}}+R_{\tau_{n}}} 1_{\left(\tau_{k} \leq t\right)}>x \mid \tau_{n}=s\right) & =\operatorname{Pr}\left(D_{t-s}>x\right) \\
& \leq \operatorname{Pr}\left(D_{T}>x\right) \\
& \sim \bar{F}(x) \int_{0}^{T} \mathrm{e}^{u \phi(\alpha)} \mathrm{d} \lambda_{u} .
\end{aligned}
$$


Thus, by further conditioning on $\mathrm{e}^{-R_{s}}$, it can be shown that, uniformly for all $t \in \Lambda_{T}$,

$$
\begin{aligned}
I_{1}(x, t) & \lesssim \int_{0-}^{t}\left(\operatorname{Pr}\left(X \mathrm{e}^{-R_{s}}>x, \mathrm{e}^{-R_{s}} \leq x^{1-\delta}\right) \int_{0}^{T} \mathrm{e}^{u \phi(\alpha)} \mathrm{d} \lambda_{u}\right) \operatorname{Pr}\left(\tau_{n} \in \mathrm{d} s\right) \\
& \leq \lambda_{T} \int_{0-}^{t} \operatorname{Pr}\left(X \mathrm{e}^{-R_{s}}>x\right) \operatorname{Pr}\left(\tau_{n} \in \mathrm{d} s\right) \\
& \sim \bar{F}(x) \lambda_{T} \int_{0-}^{t} \mathrm{e}^{s \phi(\alpha)} \operatorname{Pr}\left(\tau_{n} \in \mathrm{d} s\right) \\
& \leq \bar{F}(x) \lambda_{T} \operatorname{Pr}\left(\tau_{n} \leq t\right),
\end{aligned}
$$

where in the third step we used Lemma 4.3. Following the proof of Lemma 5.3 of Tang (2004), we have

$$
\lim _{n \rightarrow \infty} \sup _{t \in \Lambda_{T}} \frac{\mathrm{EN}_{t} 1_{\left(N_{t}>n\right)}}{\lambda_{t}}=0 .
$$

Therefore, it holds for all large $n$ and uniformly for all $t \in \Lambda_{T}$ that

$$
I_{1}(x, t) \lesssim \varepsilon \bar{F}(x) \int_{0-}^{t} \mathrm{e}^{s \phi(\alpha)} \mathrm{d} \lambda_{s}
$$

By Markov's inequality and the second relation of (4.3), it holds uniformly for all $t \in \Lambda_{T}$ that

$$
\begin{aligned}
I_{2}(x, t) & \leq x^{-(1-\delta) \alpha^{*}} \int_{0-}^{t} \mathrm{Ee}^{-\alpha^{*} R_{s}} \operatorname{Pr}\left(\tau_{n} \in \mathrm{d} s\right) \\
& \leq x^{-(1-\delta) \alpha^{*}} \int_{0-}^{t} \mathrm{e}^{s \phi\left(\alpha^{*}\right)} \mathrm{d} \lambda_{s} \\
& =o(1) \bar{F}(x) \int_{0-}^{t} \mathrm{e}^{s \phi(\alpha)} \mathrm{d} \lambda_{s} .
\end{aligned}
$$

This ends the proof.

Lemma 4.6. Let $Z$ be an exponential functional of a Lévy process $\left\{R_{t}, t \geq 0\right\}$ defined as

$$
Z=\int_{0}^{\infty} \mathrm{e}^{-R_{t}} \mathrm{~d} t
$$

Then, we have the following two results: (1) $Z<\infty$ almost surely if and only if $R_{t} \rightarrow \infty$ almost surely as $t \rightarrow \infty$;

(2) If $\alpha>0$ and $\phi(\alpha)<0$, then $\mathrm{E} Z^{\alpha}<\infty$.

Proof. See Subsection 2.1 of Maulik and Zwart (2006). 


\subsection{Proof of Theorem 2.1}

By Lemma 4.2, relation (2.4) holds for every fixed $t \in \Lambda$. We formulate the proof of the uniformity into two steps.

First, we establish the local uniformity of relation (2.4); that is, for arbitrarily fixed $T \in \Lambda$, relation (2.4) holds uniformly for all $t \in \Lambda_{T}$. For arbitrarily fixed $0<\delta<1$ and $n=1,2, \ldots$, we have

$$
\begin{aligned}
& \operatorname{Pr}\left(D_{t}>x\right) \\
\leq & \operatorname{Pr}\left(\sum_{k=1}^{n} X_{k} \mathrm{e}^{-R_{\tau_{k}}} 1_{\left(\tau_{k} \leq t\right)}>x-\delta x\right)+\operatorname{Pr}\left(\sum_{k=n+1}^{\infty} X_{k} \mathrm{e}^{-R_{\tau_{k}}} 1_{\left(\tau_{k} \leq t\right)}>\delta x\right) \\
= & I_{1}(x, t)+I_{2}(x, t) .
\end{aligned}
$$

By Lemma 4.4, it holds uniformly for all $t \in \Lambda_{T}$ that

$$
I_{1}(x, t) \lesssim(1-\delta)^{-\alpha} \bar{F}(x) \int_{0-}^{t} \mathrm{e}^{s \phi(\alpha)} \mathrm{d} \lambda_{s} .
$$

By Lemma 4.5, for arbitrarily fixed $\varepsilon>0$, it holds for all large $n$ and uniformly for all $t \in \Lambda_{T}$ that

$$
I_{2}(x, t) \lesssim \varepsilon \delta^{-\alpha} \bar{F}(x) \int_{0-}^{t} \mathrm{e}^{s \phi(\alpha)} \mathrm{d} \lambda_{s}
$$

It follows that, uniformly for all $t \in \Lambda_{T}$,

$$
\operatorname{Pr}\left(D_{t}>x\right) \lesssim\left((1-\delta)^{-\alpha}+\varepsilon \delta^{-\alpha}\right) \bar{F}(x) \int_{0-}^{t} \mathrm{e}^{s \phi(\alpha)} \mathrm{d} \lambda_{s} .
$$

By the arbitrariness of $\varepsilon$ and $\delta$, we prove that, uniformly for all $t \in \Lambda_{T}$,

$$
\operatorname{Pr}\left(D_{t}>x\right) \lesssim \bar{F}(x) \int_{0-}^{t} \mathrm{e}^{s \phi(\alpha)} \mathrm{d} \lambda_{s}
$$

The corresponding lower bound can be constructed in a similar way. Actually, by Lemma 4.4 , it holds uniformly for all $t \in \Lambda_{T}$ that

$$
\begin{aligned}
\operatorname{Pr}\left(D_{t}>x\right) & \geq \operatorname{Pr}\left(\sum_{k=1}^{n} X_{k} \mathrm{e}^{-R_{\tau_{k}}} 1_{\left(\tau_{k} \leq t\right)}>x\right) \\
& \sim \bar{F}(x) \int_{0-}^{t} \mathrm{e}^{s \phi(\alpha)}\left(\mathrm{d} \lambda_{s}-\sum_{k=n+1}^{\infty} \operatorname{Pr}\left(\tau_{k} \in \mathrm{d} s\right)\right) \\
& \geq \bar{F}(x)\left(1-\mathrm{Ee}^{\tau_{n} \phi(\alpha)}\right) \int_{0-}^{t} \mathrm{e}^{s \phi(\alpha)} \mathrm{d} \lambda_{s} .
\end{aligned}
$$

Since $\mathrm{Ee}^{\tau_{n} \phi(\alpha)}$ tends to 0 as $n \rightarrow \infty$, it follows that, uniformly for all $t \in \Lambda_{T}$,

$$
\operatorname{Pr}\left(D_{t}>x\right) \gtrsim \bar{F}(x) \int_{0-}^{t} \mathrm{e}^{s \phi(\alpha)} \mathrm{d} \lambda_{s} .
$$


Next, we extend the uniformity of relation (2.4) to $\Lambda$. For arbitrarily fixed $0<\varepsilon<1$, choose some large $T \in \Lambda$ such that

$$
\int_{T}^{\infty} \mathrm{e}^{s \phi(\alpha)} \mathrm{d} \lambda_{s} \leq \varepsilon \int_{0}^{T} \mathrm{e}^{s \phi(\alpha)} \mathrm{d} \lambda_{s}
$$

Let $t \in[T, \infty]$ and apply (4.14) and Lemma 4.2. On one hand,

$$
\operatorname{Pr}\left(D_{t}>x\right) \leq \operatorname{Pr}\left(D_{\infty}>x\right) \sim \bar{F}(x) \int_{0-}^{\infty} \mathrm{e}^{s \phi(\alpha)} \mathrm{d} \lambda_{s} \leq(1+\varepsilon) \bar{F}(x) \int_{0-}^{t} \mathrm{e}^{s \phi(\alpha)} \mathrm{d} \lambda_{s},
$$

and on the other hand,

$$
\begin{aligned}
\operatorname{Pr}\left(D_{t}>x\right) & \geq \operatorname{Pr}\left(D_{T}>x\right) \\
& \sim \bar{F}(x) \int_{0-}^{T} \mathrm{e}^{s \phi(\alpha)} \mathrm{d} \lambda_{s} \\
& \geq \frac{1}{1+\varepsilon} \bar{F}(x) \int_{0-}^{\infty} \mathrm{e}^{s \phi(\alpha)} \mathrm{d} \lambda_{s} \\
& \geq \frac{1}{1+\varepsilon} \bar{F}(x) \int_{0-}^{t} \mathrm{e}^{s \phi(\alpha)} \mathrm{d} \lambda_{s} .
\end{aligned}
$$

By these two estimates and the arbitrariness of $\varepsilon$, we see that relation (2.4) holds also uniformly for all $t \in[T, \infty]$.

\subsection{Proof of Theorem 3.1}

Substituting (3.1) into (3.2) yields that, for every $t \in \Lambda$,

$$
\psi(x, t)=\operatorname{Pr}\left(\inf _{0 \leq s \leq t}\left(x+c \int_{0}^{s} \mathrm{e}^{-R_{u}} \mathrm{~d} u-D_{s}\right)<0\right)
$$

where $0 \leq s \leq t$ is understood as $0 \leq s<\infty$ when $t=\infty$. Therefore, it follows immediately from Theorem 2.1 that, uniformly for all $t \in \Lambda$,

$$
\psi(x, t) \leq \operatorname{Pr}\left(D_{t}>x\right) \lesssim \bar{F}(x) \int_{0-}^{t} \mathrm{e}^{s \phi(\alpha)} \mathrm{d} \lambda_{s} .
$$

It remains to derive the corresponding uniform asymptotic lower bound for $\psi(x, t)$. Still start from (4.15) and let $T \in \Lambda$ be arbitrarily fixed. It holds for arbitrarily fixed $\delta>0$ and all $T \leq t \leq \infty$ that

$$
\begin{aligned}
\psi(x, t) & \geq \operatorname{Pr}\left(D_{t}-c \int_{0}^{\infty} \mathrm{e}^{-R_{u}} \mathrm{~d} u>x\right) \\
& \geq \operatorname{Pr}\left(D_{t}>(1+\delta) x\right)-\operatorname{Pr}\left(c \int_{0}^{\infty} \mathrm{e}^{-R_{u}} \mathrm{~d} u>\delta x\right) .
\end{aligned}
$$

By Theorem 2.1, it holds uniformly for all $t \in \Lambda$ that

$$
\operatorname{Pr}\left(D_{t}>(1+\delta) x\right) \sim(1+\delta)^{-\alpha} \bar{F}(x) \int_{0-}^{t} \mathrm{e}^{s \phi(\alpha)} \mathrm{d} \lambda_{s} .
$$


By Markov's inequality,

$$
\operatorname{Pr}\left(c \int_{0}^{\infty} \mathrm{e}^{-R_{u}} \mathrm{~d} u>\delta x\right) \leq\left(\frac{\delta x}{c}\right)^{-\alpha^{*}} \mathrm{E}\left(\int_{0}^{\infty} \mathrm{e}^{-R_{u}} \mathrm{~d} u\right)^{\alpha^{*}},
$$

where the finiteness of $\mathrm{E}\left(\int_{0}^{\infty} \mathrm{e}^{-R_{u}} \mathrm{~d} u\right)^{\alpha^{*}}$ is guaranteed by Lemma 4.6. Substituting these two estimates into (4.16) and using the arbitrariness of $\delta$, we see that, uniformly for all $T \leq t \leq \infty$ that

$$
\psi(x, t) \gtrsim \bar{F}(x) \int_{0-}^{t} \mathrm{e}^{s \phi(\alpha)} \mathrm{d} \lambda_{s}
$$

We now focus on the uniformity of (4.17) over $t \in \Lambda_{T}$. Clearly,

$$
\begin{aligned}
\psi(x, t) & \geq \operatorname{Pr}\left(D_{\tau_{N_{t}}}-c \int_{0}^{\tau_{N_{t}}} \mathrm{e}^{-R_{u}} \mathrm{~d} u>x\right) \\
& =\operatorname{Pr}\left(\sum_{k=1}^{\infty}\left(X_{k} \mathrm{e}^{-R_{\tau_{k}}}-c \int_{\tau_{k-1}}^{\tau_{k}} \mathrm{e}^{-R_{u}} \mathrm{~d} u\right) 1_{\left(\tau_{k} \leq t\right)}>x\right) .
\end{aligned}
$$

It follows that, for arbitrarily fixed $\delta>0$ and $n=1,2, \ldots$,

$$
\begin{aligned}
\psi(x, t) \geq & \operatorname{Pr}\left(\sum_{k=1}^{n} X_{k} \mathrm{e}^{-R_{\tau_{k}}} 1_{\left(\tau_{k} \leq t\right)}>(1+2 \delta) x\right)-\operatorname{Pr}\left(c \sum_{k=1}^{n} \int_{\tau_{k-1}}^{\tau_{k}} \mathrm{e}^{-R_{u}} \mathrm{~d} u 1_{\left(\tau_{k} \leq t\right)}>\delta x\right) \\
& \quad-\operatorname{Pr}\left(c \sum_{k=n+1}^{\infty} \int_{\tau_{k-1}}^{\tau_{k}} \mathrm{e}^{-R_{u}} \mathrm{~d} u 1_{\left(\tau_{k} \leq t\right)}>\delta x\right) \\
= & I_{1}(x, t)-I_{2}(x, t)-I_{3}(x, t) .
\end{aligned}
$$

By Lemma 4.4, it holds uniformly for all $t \in \Lambda_{T}$ that

$$
\begin{aligned}
I_{1}(x, t) & \sim(1+2 \delta)^{-\alpha} \bar{F}(x) \int_{0-}^{t} \mathrm{e}^{s \phi(\alpha)} \sum_{k=1}^{n} \operatorname{Pr}\left(\tau_{k} \in \mathrm{d} s\right) \\
& \geq(1+2 \delta)^{-\alpha}\left(1-\operatorname{Ee}^{\tau_{n} \phi(\alpha)}\right) \bar{F}(x) \int_{0-}^{t} \mathrm{e}^{s \phi(\alpha)} \mathrm{d} \lambda_{s} .
\end{aligned}
$$

Introduce a random variable $Z$ independent of $\left\{R_{t}, t \geq 0\right\},\left\{N_{t}, t \geq 0\right\}$ and equal in distribution to $\int_{0}^{\infty} \mathrm{e}^{-R_{t}} \mathrm{~d} t$. By Lemma 4.6, $\mathrm{E} Z^{\alpha^{*}}<\infty$. Hence, by Markov's inequality and the second relation of (4.3), $\operatorname{Pr}(Z>x)=o(\bar{F}(x))$. It holds uniformly for all $t \in \Lambda_{T}$ that

$$
\begin{aligned}
I_{2}(x, t) & \leq \sum_{k=1}^{n} \operatorname{Pr}\left(\int_{\tau_{k-1}}^{\tau_{k}} \mathrm{e}^{-R_{u}} \mathrm{~d} u 1_{\left(\tau_{k} \leq t\right)}>\frac{\delta x}{c n}\right) \\
& \leq \int_{0-}^{t} \operatorname{Pr}\left(\int_{0}^{s} \mathrm{e}^{-R_{u}} \mathrm{~d} u>\frac{\delta x}{c n}\right) \sum_{k=1}^{n} \operatorname{Pr}\left(\tau_{k} \in \mathrm{d} s\right) \\
& \leq \operatorname{Pr}\left(Z>\frac{\delta x}{c n}\right) \sum_{k=1}^{n} \operatorname{Pr}\left(\tau_{k} \leq t\right) \\
& =o(1) \bar{F}(x) \int_{0-}^{t} \mathrm{e}^{s \phi(\alpha)} \mathrm{d} \lambda_{s} .
\end{aligned}
$$


For arbitrarily fixed $0<\varepsilon<1$, further introduce a nonnegative random variable $Z_{\varepsilon}$ independent of $\left\{R_{t}, t \geq 0\right\},\left\{N_{t}, t \geq 0\right\}$ and with tail given by

$$
\operatorname{Pr}\left(Z_{\varepsilon}>x\right)=\varepsilon \bar{F}(x) \vee \operatorname{Pr}(Z>x), \quad x \geq 0 .
$$

Hence, $\operatorname{Pr}\left(Z_{\varepsilon}>x\right) \sim \varepsilon \bar{F}(x)$ and the distribution of $Z_{\varepsilon}$ belongs to the class $\mathcal{R}_{-\alpha}$ too. Clearly,

$$
\begin{aligned}
I_{3}(x, t) & \leq \operatorname{Pr}\left(c \mathrm{e}^{-R_{\tau_{n}}} 1_{\left(\tau_{n} \leq t\right)} \sum_{k=n+1}^{\infty} \int_{\tau_{k-1}}^{\tau_{k}} \mathrm{e}^{-\left(R_{u}-R_{\tau_{n}}\right)} \mathrm{d} u>\delta x\right) \\
& =\operatorname{Pr}\left(Z \mathrm{e}^{-R_{\tau_{n}}} 1_{\left(\tau_{n} \leq t\right)}>\frac{\delta x}{c}\right) \\
& \leq \operatorname{Pr}\left(Z_{\varepsilon} \mathrm{e}^{-R_{\tau_{n}}} 1_{\left(\tau_{n} \leq t\right)}>\frac{\delta x}{c}\right) .
\end{aligned}
$$

By Lemma 4.3, it holds uniformly for all $t \in \Lambda_{T}$ that

$$
I_{3}(x, t) \lesssim \operatorname{Pr}\left(Z_{\varepsilon}>\frac{\delta x}{c}\right) \int_{0-}^{t} \mathrm{e}^{s \phi(\alpha)} \operatorname{Pr}\left(\tau_{n} \in \mathrm{d} s\right) \sim \varepsilon c^{\alpha} \delta^{-\alpha} \bar{F}(x) \int_{0-}^{t} \mathrm{e}^{s \phi(\alpha)} \mathrm{d} \lambda_{s}
$$

Substituting these estimates into (4.18), we obtain that, uniformly for all $t \in \Lambda_{T}$,

$$
\psi(x, t) \gtrsim\left((1+2 \delta)^{-\alpha}\left(1-\mathrm{Ee}^{\tau_{n} \phi(\alpha)}\right)-\varepsilon c^{\alpha} \delta^{-\alpha}\right) \bar{F}(x) \int_{0-}^{t} \mathrm{e}^{s \phi(\alpha)} \mathrm{d} \lambda_{s} .
$$

By the arbitrariness of $\delta, \varepsilon$, and $n$, relation (4.17) holds uniformly for all $t \in \Lambda_{T}$.

Acknowledgments. The authors would like to thank the anonymous referee for his/her careful reading and useful comments. Qihe Tang acknowledges support of the 2008 Old Gold Summer Fellowship from the University of Iowa, Guojing Wang acknowledges support of the Natural Science Foundation of Jiangsu Province (Grant No: KB2008155), and Kam C. Yuen acknowledges support of a research grant from the University of Hong Kong.

\section{References}

[1] Applebaum, D. Lévy Processes and Stochastic Calculus. Cambridge University Press, Cambridge, 2004.

[2] Asmussen, S. Ruin Probabilities. World Scientific, London, 2003.

[3] Bingham, N. H.; Goldie, C. M.; Teugels, J. L. Regular Variation. Cambridge University Press, Cambridge, 1987.

[4] Björk, T. Arbitrage Theory in Continuous Time. Oxford University Press, New York, 1998.

[5] Breiman, L. On some limit theorems similar to the arc-sin law. Theory Prob. Appl. 10 (1965) 323-331. 
[6] Cai, J. Ruin probabilities and penalty functions with stochastic rates of interest. Stochastic Process. Appl. 112 (2004), no. 1, 53-78.

[7] Chen, Y.; Yuen, K. C. Sums of pairwise quasi-asymptotically independent random variables with consistent variation. Stoch. Models 25 (2009), no. 1, 76-89.

[8] Cont, R.; Tankov, P. Financial Modelling with Jump Processes. Chapman \& Hall/CRC, Boca Raton, FL, 2004.

[9] Emmer, S.; Klüppelberg, C. Optimal portfolios when stock prices follow an exponential Lévy process. Finance Stoch. 8 (2004), no. 1, 17-44.

[10] Emmer, S.; Klüppelberg, C.; Korn, R. Optimal portfolios with bounded capital at risk. Math. Finance 11 (2001), no. 4, 365-384.

[11] Frolova, A.; Kabanov, Y.; Pergamenshchikov, S. In the insurance business risky investments are dangerous. Finance Stoch. 6 (2002), no. 2, 227-235.

[12] Goovaerts, M. J.; Kaas, R.; Laeven, R. J. A.; Tang, Q.; Vernic, R. The tail probability of discounted sums of Pareto-like losses in insurance. Scand. Actuar. J. (2005), no. 6, 446-461.

[13] Heyde, C. C.; Wang, D. Finite-time ruin probability with an exponential Lévy process investment return and heavy-tailed claims. Adv. in Appl. Probab. 41 (2009), no. 1, 206-224.

[14] Kalashnikov, V.; Norberg, R. Power tailed ruin probabilities in the presence of risky investments. Stochastic Process. Appl. 98 (2002), no. 2, 211-228.

[15] Klüppelberg, C.; Kostadinova, R. Integrated insurance risk models with exponential Lévy investment. Insurance Math. Econom. 42 (2008), no. 2, 560-577.

[16] Kostadinova, R. Optimal investment for insurers when the stock price follows an exponential Lévy process. Insurance Math. Econom. 41 (2007), no. 2, 250-263.

[17] Maulik, K.; Zwart, B. Tail asymptotics for exponential functionals of Lévy processes Stochastic Process. Appl. 116 (2006), no. 2, 156-177.

[18] Paulsen, J. Risk theory in a stochastic economic environment. Stochastic Process. Appl. 46 (1993), no. 2, 327-361.

[19] Paulsen, J. On Cramér-like asymptotics for risk processes with stochastic return on investments. Ann. Appl. Probab. 12 (2002), no. 4, 1247-1260.

[20] Paulsen, J. Optimal dividend payouts for diffusions with solvency constraints. Finance Stoch. 7 (2003), no. 4, 457-473.

[21] Paulsen, J. Ruin models with investment income. Probab. Surv. 5 (2008), 416-434.

[22] Paulsen, J.; Gjessing, H. K. Ruin theory with stochastic return on investments. Adv. in Appl. Probab. 29 (1997), no. 4, 965-985. 
[23] Protter, P. E. Stochastic Integration and Differential Equations. Second edition. Springer-Verlag, Berlin, 2005.

[24] Resnick, S. I.; Willekens, E. Moving averages with random coefficients and random coefficient autoregressive models. Comm. Statist. Stochastic Models 7 (1991), no. 4, $511-525$.

[25] Sato, K. Lévy Processes and Infinitely Divisible Distributions. Cambridge University Press, Cambridge, 1999.

[26] Schmidli, H. On minimizing the ruin probability by investment and reinsurance. Ann. Appl. Probab. 12 (2002), no. 3, 890-907.

[27] Tang, Q. Uniform estimates for the tail probability of maxima over finite horizons with subexponential tails. Probab. Engrg. Inform. Sci. 18 (2004), no. 1, 71-86.

[28] Tang, Q.; Tsitsiashvili, G. Precise estimates for the ruin probability in finite horizon in a discrete-time model with heavy-tailed insurance and financial risks. Stochastic Process. Appl. 108 (2003), no. 2, 299-325.

[29] Wang, D.; Tang, Q. Tail probabilities of randomly weighted sums of random variables with dominated variation. Stoch. Models 22 (2006), no. 2, 253-272.

[30] Wang, G.; Wu, R. Distributions for the risk process with a stochastic return on investments. Stochastic Process. Appl. 95 (2001), no. 2, 329-341.

[31] Willekens, E. On the supremum of an infinitely divisible process. Stochastic Process. Appl. 26 (1987), no. 1, 173-175.

[32] Yuen, K. C.; Wang, G.; Ng, K. W. Ruin probabilities for a risk process with stochastic return on investments. Stochastic Process. Appl. 110 (2004), no. 2, 259-274.

[33] Yuen, K. C.; Wang, G.; Wu, R. On the renewal risk process with stochastic interest. Stochastic Process. Appl. 116 (2006), no. 10, 1496-1510.

[34] Zhang, Y.; Shen, X.; Weng, C. Approximation of the tail probability of randomly weighted sums and applications. Stochastic Process. Appl. 119 (2009), no. 2, 655-675. 